\title{
From atomic physics, to upper-atmospheric chemistry, to cosmology: A "laser photometric ratio star" to calibrate telescopes at major observatories
}

\author{
Justin Albert $^{1}$, Dmitry Budker ${ }^{2}$, and Hossein Sadeghpour ${ }^{3}$ \\ ${ }^{1}$ University of Victoria \\ ${ }^{2}$ Johannes Gutenberg Universität Mainz \\ ${ }^{3}$ Center for Astrophysics | Harvard \& Smithsonian
}

March 4, 2022

\begin{abstract}
This Research Highlight showcases the two Research Papers entitled, A precise photometric ratio via laser excitation of the sodium layer - I. One-photon excitation using $342.78 \mathrm{~nm}$ light, https://doi.org/10.1093/mnras/stab1621 and A precise photometric ratio via laser excitation of the sodium layer - II. Two-photon excitation using lasers detuned from $589.16 \mathrm{~nm}$ and $819.71 \mathrm{~nm}$ resonances, https://doi.org/10.1093/mnras/stab1619.
\end{abstract}

\section{Hosted file}

NaturalSciencesWileyLPRSHighlight_16feb22.pdf available at https://authorea.com/users/456594/ articles/553588-from-atomic-physics-to-upper-atmospheric-chemistry-to-cosmology-a-laserphotometric-ratio-star-to-calibrate-telescopes-at-major-observatories

[1] A. G. Riess, et al., Astron. J. 1998, 116, 1009.

[2] S. Perlmutter, et al., Astrophys. J. 1999, 517, 565.

[3] J. P. Gardner, et al., Space Sci. Rev. 2006, 123, 485; and https://jwst.nasa.gov.

[4] Ž. Ivezić, et al., Astrophys. J. 2019, 873, 111; and https://www.lsst.org.

[5] a) D. O. Jones, et al., Astrophys. J. 2018, 857, 51; b) M. Betoule, et al., Astron. Astrophys. 2014, 568, A22; c) W. M. Wood-Vasey, et al., Astrophys. J. 2007, 666, 694.

[6] For projects to provide upcoming improvement on the precision of measurements of absolute magnitude values of astronomical sources, rather than on the precision of measurements of ratios of such magnitudes as functions of color, please additionally see, for example:
i) ALTAIR Collaboration 2022 (http://projectaltair.org);
ii ) ORCASat Collaboration 2022 (https://orcasat.ca); and,
iii ) ORCAS Project 2022 (https://asd.gsfc.nasa.gov/orcas/).

[7] C. W. Stubbs, J. L. Tonry, Astrophys. J. 2006, 646, 1436.

[8] Image from L. Michaille, J. C. Quartel, J. C. Dainty, N. J. Wooder, R. W. Wilson, T. Gregory, ING Newsletter 2000, 2, 1. 
[9] Please see, for example: a)S. S. Olivier, C. E. Max, in Laser Guide Star Adaptive Optics: Present and Future, IAU Symp. 158 (Eds: J. G. Robertson, W. J. Tango), Kluwer Press, Dordrecht, Germany 1994, p. 283; b) D. Bonaccini, et al., in Adaptive Optics Systems and Technology II, Proc. SPIE 4494 (Eds: R. K. Tyson, D. Bonaccini, M. C. Roggemann), SPIE Digital Library, Bellingham, WA, USA 2002, p. 276; c) P. L. Wizinowich, et al., Publ. Astron. Soc. Pac.2006, 118, 297.

[10] a) V.M. Slipher, Popular Astron. 1929, 37, 327; b) S. Chapman, Astrophys. J. 1939, 90, 309.

[11] J. M. C. Plane, W. Feng, E. C. M. Dawkins, Chem. Rev.2015, 115, 4497.

[12] a) P. Juncar, J. Pinard, J. Harmon, A. Chartier, Metrologia 1981, 17, 77; b) D. E. Kelleher, L. I. Podobedova, J. Phys. Chem. Ref. Data 2008, 37, 267; c) A. Kramida, Y. Ralchenko, J. Reader, et al., Technical report, Atomic Spectra Database (ver. 5.8), NIST 2020, available at: https://physics.nist. gov/asd.

[13] Please see, for example: a) J. Albert, Astron. J. 2012, 143, 8; b) J. B. Holberg, P. Bergeron, Astrophys. J. 2006, 132, 1221; c) R. C. Bohlin, R. L. Gilliland, Astron. J. 2004, 127, 350.

[14] a) J. E. Albert, D. Budker, K. Chance, I. E. Gordon, F. Pedreros Bustos, M. Pospelov, S.M. Rochester, H. R. Sadeghpour, Mon. Not. R. Astron. Soc. 2021, 508, 4399 [arXiv:2001.10958]; b) J. E. Albert, D. Budker, K. Chance, I. E. Gordon, F. Pedreros Bustos, M. Pospelov, S. M.Rochester, H. R. Sadeghpour, Mon. Not. R. Astron. Soc. 2021, 508, 4412 [arXiv:2010.08683].

[15] U. Gaubatz, P. Rudecki, S. Schiemann, K. Bergmann, J. Chem. Phys. 1990, 92, 5363.

[16] a) K. Bergmann, N. V. Vitanov, B. W. Shore, J. Chem. Phys. 2015, 142, 170901; b) N. V. Vitanov, A. A. Rangelov, B. W. Shore, K. Bergmann, Rev. Mod.Phys. 2017, 89, 015006.

[17] Please see, for example: M. Turner, Phys. Today 2003, 56, 10.

[18] a) S. Weinberg, Rev. Mod. Phys. 1989, 61, 1; b) P. J. E. Peebles, B.Ratra, Rev. Mod Phys. 2003, 75, 559 .

[19] Please see, for example: C.-I. Chiang, J. M. Leedom, H. Murayama, Phys. Rev. D 2019, 100, 043505. 